\title{
A hierarchical self-healing SLA for cloud computing
}

\begin{abstract}
The service level agreement (SLA) is a mutual contract between the service provider and consumer which determines the agreed service level objective (SLO). The common SLA is a plain documental agreement without any relation to other dependent SLAs during the different layers of cloud computing. Hence, the cloud computing environment needs the hierarchical and autonomic SLA. This paper proposes the SH-SLA model to generate a hierarchical self-healing SLA in cloud computing. The self-healing ability contains the SLA monitoring, violation detecting and violation reacting processes. In SH-SLA, the related SLAs communicate with each other hierarchically. The SLA would be able to check its QoS and notify the recent status to dependent SLAs. Furthermore, SH-SLA could prevent or propagate the notified violations by an urgent reaction. Consequently, the service providers have a great chance to prevent the violated SLA before sensing by end users. The SH-SLA model is simulated and the experiment results have presented the violation detection and reaction abilities of the proposed model in cloud computing. Besides, the end users meet the lesser violations in SH-SLA than the common SLA.
\end{abstract}

Keyword: Service level agreement; Cloud computing; Self monitoring SLA; Hierarchical SLA; Self-healing SLA 